\title{
Frauen in der Mathematik
}

\author{
Thomas Vogt im Gespräch mit Marie-Françoise Roy
}

\begin{abstract}
Seit Jahrzehnten engagiert sich Marie-Françoise Roy neben ihrer Forschung und Lehre in Algebraischer Geometrie auch für die Frauenrechte in der Mathematik. Dabei lässt sich die inzwischen emeritierte Professorin der Universität Rennes nicht nur von ihren Beobachtungen in ihrem professionellen Umfeld leiten, sondern versucht systematisch zu erfassen, welche Probleme es genau gibt und wie sie sich möglicherweise aus der Welt schaffen lassen. Jüngstes Beispiel für ihre Arbeit und eines der größten Projekte, das sie koordiniert hat, ist eine Umfrage unter 32 ooo Wissenschaftlerinnen und Wissenschaftlern weltweit zur Situation von Frauen in der Forschung namens „Gender Gap in Science - A Global Approach to the Gender Gap in Mathematical, Computing, and Natural Sciences: How to Measure It, How to Reduce It?" Kürzlich wurden die ersten Ergebnisse publiziert, was die DMV-Mitteilungen zum Anlass nahmen, mit der engagierten Mathematik-Professorin zu sprechen.
\end{abstract}

Die NGO „Verein für Frauen in der Mathematik“ wurde in den Vereinigten Staaten vor fast 50 Jahren (1971) gegründet. EWM, das europäisches Pendant dazu, wurde von einer Gruppe gegründet, der Sie 1986 angehörten ...

Es gibt weitere regionale Organisationen für Frauen in der Mathematik, wie IWM, also Indian Women in Mathematics, welche 2010 gegründet wurde, oder AWMA, die African Women in Mathematics Association, gegründet 2013. Darüber hinaus haben sich in letzter Zeit viele Aktivitäten in lateinamerikanischen Ländern entwickelt, insbesondere in Brasilien, Chile und Mexiko. CWM, das Komitee der Internationalen Mathematischen Union für Frauen in der Mathematik, dessen Vorsitz ich innehabe, listet auf seiner Website mehr als 35 verschiedene Länder auf, in denen es spezifische Initiativen für Frauen in der Mathematik gibt; entweder unabhängige Organisationen oder Komitees nationaler mathematischer Gesellschaften.

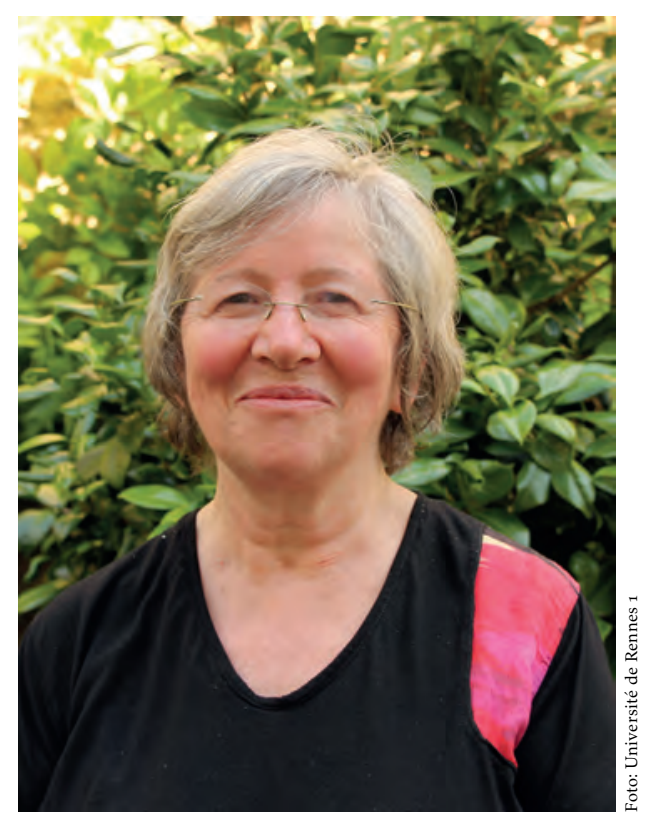

Marie-Françoise Roy
Der Internationale Tag der Frauen in der Mathematik wurde zum ersten Mal am 12. Mai 2019 begangen.

Offiziell heißt die Initiative „12. Mai zur Feier von Frauen in der Mathematik", und es ist kein offizieller internationaler Tag in der Liste der UNO. Tatsächlich haben wir jetzt einen offiziellen Internationalen Tag der Mathematik (International Day of Mathematics, IDM), und zwar am 14. März (Pi-Tag), der von der UNESCOGeneralversammlung beschlossen und im Jahr 2020 eingeführt wurde [siehe Interview in Heft 1-2020, Anm. d. Redakt.]. Ein ausgeglichenes Geschlechterverhältnis ist eines der Ziele des IDM. Der 12. Mai ist übrigens der Geburtstag der iranischen Mathematikerin Maryam Mirzakhani, der ersten und einzigen weiblichen FieldsMedaillengewinnerin, die leider sehr jung verstorben ist. Aus diesem Grund wurde auf dem ersten Welttreffen für Frauen in der Mathematik (CWM) in Rio auf Vorschlag des Frauenkomitees der Iranischen Mathematischen Gesellschaft die weltweite Feier für Frauen in der Mathematik am 12. Mai beschlossen. Der 12. Mai ist übrigens auch das Geburtsdatum von Florence Nightingale, der britischen Sozialreformerin, Statistikerin und Begründerin der modernen Krankenpflege. Und am 12. Mai 1819 schrieb die französische Mathematikerin Sophie Germain einen berühmten Brief an Karl Friedrich Gauß über ihren Plan zum Beweis von Fermats letztem Satz.

Bei der diesjährigen 12. Mai-Feier ist die Zahl der Veranstaltungen von 110 im letzten Jahr auf 140 in diesem Jahr gestiegen und das trotz der Unsicherheiten im $\mathrm{Zu}-$ sammenhang mit der Corona-Pandemie. Viele dieser Veranstaltungen fanden online statt.

\section{Können Sie bitte ein Beispiel nennen?}

Die Koordinationsgruppe vom 12. Mai hatte eine Vereinbarung mit zalafilms, so dass wir kostenlose Vorführungen des wundervollen Films Secrets of the Surface für alle anbieten konnten, die sich auf unserer Website angemeldet haben. Dieser einfühlsame Film von George Csicsery beschreibt die mathematische Vision von Maryam Mirzakhani. Die Resonanz war mit mehr als 20 ooo Anmel- 


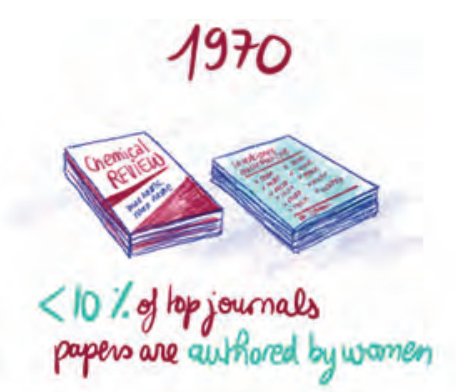

dungen aus 131 verschiedenen Ländern überwältigend. Dank mehrerer Freiwilliger konnten Untertitel in mehreren Sprachen angeboten werden, z. B. Italienisch, Portugiesisch, Spanisch und Türkisch, und die bereits von zalafilms angebotenen Untertitel in Farsi und Französisch konnten verbessert werden. Maryam Mirzakhani ist im Iran eine Ikone, weit über die mathematische Gemeinschaft hinaus; dies spiegelt sich auch in der Tatsache wider, dass mehr als 1/3 der Anmeldungen aus dem Iran kamen.

Warum ist „Frauen in der Mathematik" immer noch ein so großes Thema?

Das hängt sehr von den Indikatoren ab, die Sie betrachten. Wenn Sie den Anteil weiblicher Autoren in der Mathematik weltweit betrachten, so gibt es in den letzten 50 Jahren einen kontinuierliche Anstieg; derzeit sind etwa 30\% der Autoren von mathematischen Arbeiten weiblich.

Aber wenn Sie die Publikationen in den Spitzenzeitschriften anschauen, sieht die Situation ganz anders aus: Trotz des wachsenden Frauenanteils unter den Autor ${ }^{\star}$ innen stagniert der Frauenanteil in Top-Zeitschriften. Darüber hinaus ist die Situation in der Mathematik und in der theoretischen Physik viel schlechter als in anderen Disziplinen wie Astronomie, Astrophysik oder Chemie, wo eine positive Veränderung zu beobachten ist.

\section{Wie kommen Sie zu dieser Einschätzung?}

Das ist eines der Ergebnisse unseres jüngsten Forschungsprojekts [1] zur ,Geschlechterlücke in der Wissenschaft: Wie man sie messen und wie man sie verringern kann'.

Die Geschlechterlücke beschreibt den Unterschied zwischen Frauen und Männern ,in Bezug auf den Grad ihrer Teilhabe, ihres Zugangs, ihrer Rechte, ihres Lohns und ihrer Leistungen". Nach statistischen Angaben der UNESCO sind weniger als $30 \%$ der weltweiten Forscher*innen weiblich, was die Existenz einer deutlichen Geschlechterlücke in der Wissenschaft zeigt. Um diese Lücke zu verstehen und zu verringern, ist es notwendig, die verschiedenen Faktoren zu identifizieren, die Frauen davon abhalten, eine Karriere in der Wissenschaft einzuschlagen.

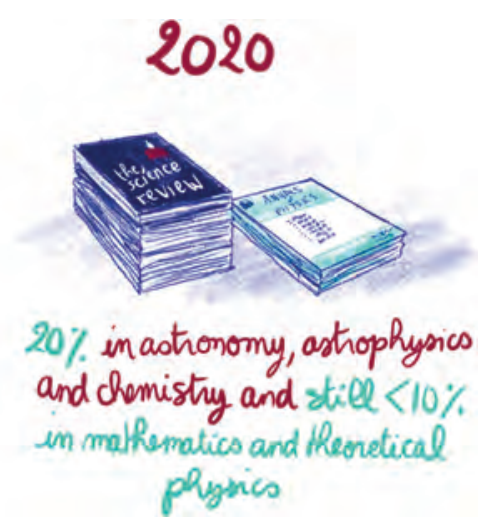

Bitte erzählen Sie uns noch mehr von Ihrem Forschungsprojekt zur Geschlechterlücke in der Wissenschaft.

Unser Projekt trägt aus drei sich ergänzenden Perspektiven zur Analyse bei:

- Die globale Umfrage unter Wissenschaftler*innen befasst sich mit Fragen zu fehlenden Vorbildern, zu Ausgrenzungsgefühlen, zu Belästigung, niedriger Teilnahme und Vorbehalten.

- Die Studie zum Publikationsmuster liefert über die Autorschaft Erkenntnisse zum Frauenanteil in der Forschung und zu den Autor*innen in renommierten Zeitschriften.

- Die Datenbank guter Praxisbeispiele bietet den konzeptionellen Rahmen für deren Analyse, um ihre Effektivität und Wirkung zu offenbaren.

Um es kurz zu machen möchte ich sagen, dass an der Umfrage weltweit 32 ooo Wissenschaftler*innen teilgenommen haben, von denen $50 \%$ männlich und $50 \%$ weiblich waren. Die Ergebnisse bestätigen, dass die Geschlechterlücke in der Wissenschaft äußerst real ist: Sie besteht über alle Regionen, Disziplinen und Karrierestufen hinweg. Die Erfahrungen von Frauen sowohl im Ausbildungs- als auch im Beschäftigungsbereich sind durchweg weniger positiv als die der Männer:

- Über ein Viertel der Frauen gab an, persönlich sexuelle Belästigung in der Schule oder am Arbeitsplatz erlebt zu haben. Frauen gaben mehr als 14 Mal häufiger als Männer an, persönlich belästigt worden zu sein.

- Es besteht weiterhin ein Lohngefälle zwischen Frauen und Männern. Die Elternschaft hat signifikant unterschiedliche Auswirkungen auf das Leben von Frauen im Vergleich zu Männern.

Für weitere Einzelheiten möchte ich auf unsere Projektseiten gender-gap-in-science.org verweisen (siehe auch [1]).

Was ist das Besondere an Ihrer Studie im Vergleich zu anderen oder bisherigen?

Unsere Studie über Publikationsmuster ist wichtig, weil erfolgreiche akademische Karrieren stark an eine pro- 
duktive wissenschaftliche Bilanz gebunden sind und wissenschaftliche Publikationen eine Schlüsselrolle für die wissenschaftliche Reputation spielen. Das Verständnis der Publikationspraktiken in verschiedenen Disziplinen ist von größter Bedeutung.

Indem wir frühere Studien in Mathematik um Astronomie, Theoretische Physik und teilweise auch Chemie erweitert haben, konnten wir Millionen von Publikationen von 1970 bis heute analysieren. Wegen ihrer Spezifität und Vollständigkeit wählten wir zbMATH, ADS und arXiv als Datenquellen.

Unsere Ergebnisse geben einen tiefen Einblick in die Dynamik des wissenschaftlichen Publizierens: Der Frauenanteil bei den Publikationen in den Disziplinen ist stetig gestiegen. Allerdings stagniert der Frauenanteil in Spitzenzeitschriften in Mathematik und Theoretischer Physik und liegt unverändert bei etwa 10\%, während er in Astronomie und Chemie gestiegen ist. Wir stellen fest, dass es in den theoretischen Disziplinen und Teildisziplinen weniger Autorinnen gibt, während in den angewandten und den gemeinschaftlichen Bereichen ein größerer Frauenanteil zu verzeichnen ist.

In unserer Datenbank für gute Praxis haben wir eine Auswahl von Initiativen zur Verringerung der Geschlechterlücke in vielen Ländern und Disziplinen zusammengestellt, Parameter entwickelt, die gute Praxis charakterisieren, und diese auf alle Programme angewandt, um zu erklären, warum sie „funktionieren“.

Gibt es eine Geschlechterlücke in Mathematik und den Naturwissenschaften in allen Ländern der Welt gleichermaßen?

In unserem Projekt haben wir regionale Unterschiede und Unterschiede zwischen mehr und weniger entwickelten Ländern sehr genau untersucht. Und unsere wichtigste Schlussfolgerung ist, dass die Situation überall sehr ähnlich ist. In allen Regionen, in allen eingangs genannten Disziplinen und auf allen Entwicklungsstufen berichten Frauen deutlich häufiger als Männer über Diskriminierung aufgrund ihres Geschlechts. Frauen berichten auch seltener als Männer, von Kollegen respektvoll behandelt zu werden. Bei der Untersuchung nach Beschäftigungssektoren berichten Personen, die in der Industrie, in Nichtregierungsorganisationen und in Primar- und Sekundarschulen tätig sind, häufiger von einer respektvollen Behandlung durch Kollegen als jene, die im akademischen Bereich oder im staatlichen Sektor arbeiten.

Was kann, was sollte in den kommenden Jahren getan werden?

Wir haben sorgfältig Empfehlungen für den ausbildenden Bereich, für Eltern und für lokale Organisationen sowie für Gewerkschaften weltweit zusammengestellt. Mit lokalen Organisationen meinen wir alle Arten wissenschaftlicher oder pädagogischer Einrichtungen im Wissenschaftsbetrieb, in Konferenzzentren, in der industriellen Forschung u. a. Hier ist, was wir empfehlen:
- Fördern Sie eine respektvolle, kollegiale Arbeitsatmosphäre. Beobachten Sie die Unterstützung, das Wohlergehen und die Betreuung von Akademikerinnen.

- Erstellen Sie einen Leitfaden, um sexuelle Belästigung und Diskriminierung im beruflichen Umfeld zu verhindern, zu melden und dagegen vorzugehen.

- Setzen Sie sich mit den Auswirkungen der Elternschaft auf die berufliche Laufbahn von Frauen auseinander. Führen Sie bei Kandidat ${ }^{\star}$ nnen in Einstellungs- und Beförderungsprozessen eine angemessene Berücksichtigung von Kinderbetreuungszeiten (18 Monate pro Kind empfohlen) ein. In der Praxis gilt dies hauptsächlich für Frauen.

- Gewähren Sie ggf. ein reines Forschungsjahr nach dem Mutterschafts- oder Erziehungsurlaub. Erkennen und akzeptieren Sie die Existenz von diskontinuierlichen Karrieren und familiären Verpflichtungen und berücksichtigen Sie diese in Ihrer Einstellungs- und Finanzierungspolitik.

○ Gewährleisten Sie Transparenz bezüglich Gehältern, Lehrdeputat, Boni, Einstellung, Förderung und Beförderung und beobachten sie Fortschritte oder Schwierigkeiten von Wissenschaftlerinnen.

- Versuchen Sie, geschlechtsspezifische Gehaltsunterschiede zu verringern. Gewährleisten Sie, dass Frauen und Männer in Einstellungsgremien und bei Weiterbildungsmaßnahmen paritätisch vertreten sind und auch bezüglich unbewusster Voreingenommenheit geschult werden. Machen Sie Gleichstellungs- und Geschlechterfragen zur Aufgabe einer engagierten Person.

- Heißen Sie Familien willkommen und schaffen Sie eine kinderfreundliche Umgebung. Unterstützen Sie Eltern. Weisen Sie Eltern ein angemessenes Lehrdeputat zu. Kümmern Sie sich bei Konferenzen um die Bedürfnisse von Personen, die mit Kindern teilnehmen, und statten Sie die Familienzimmer in den Gästehäusern so aus, dass alle Grundbedürfnisse erfüllt sind (z. B. mit Kinderspielzeug, Hochstühlen und Wickeltischen für Babys).

- Institutionalisieren Sie die Gleichstellung der Geschlechter. Bestimmen Sie eine Person oder eine Abteilung, die innerhalb der Organisation für Genderfragen und Gleichstellung zuständig ist, und achten Sie auf ein ausgewogenes Geschlechterverhältnis bei allen Aktivitäten. Führen Sie Initiativen zur Förderung von Frauen ein. Beziehen Sie Männer in die Identifizierung von Barrieren und deren Beseitigung ein. Verstöße gegen Gleichstellungspläne sollten - zum Beispiel finanzielle - Konsequenzen haben.

- Zielen Sie bei Öffentlichkeitsarbeit und Bildungsprogrammen auf die Verkleinerung der Geschlechterlücke. Passen Sie solche Programme entsprechend an und bewerten Sie ihre Wirksamkeit. Entwickeln Sie bei künftigen Lehrpersonen ein geschlechtsspezifisches Bewusstsein und bieten Sie Schulungen in kritischem Denken an.

Brauchen wir eine Quote auf bestimmten Ebenen im akademischen Bereich? 


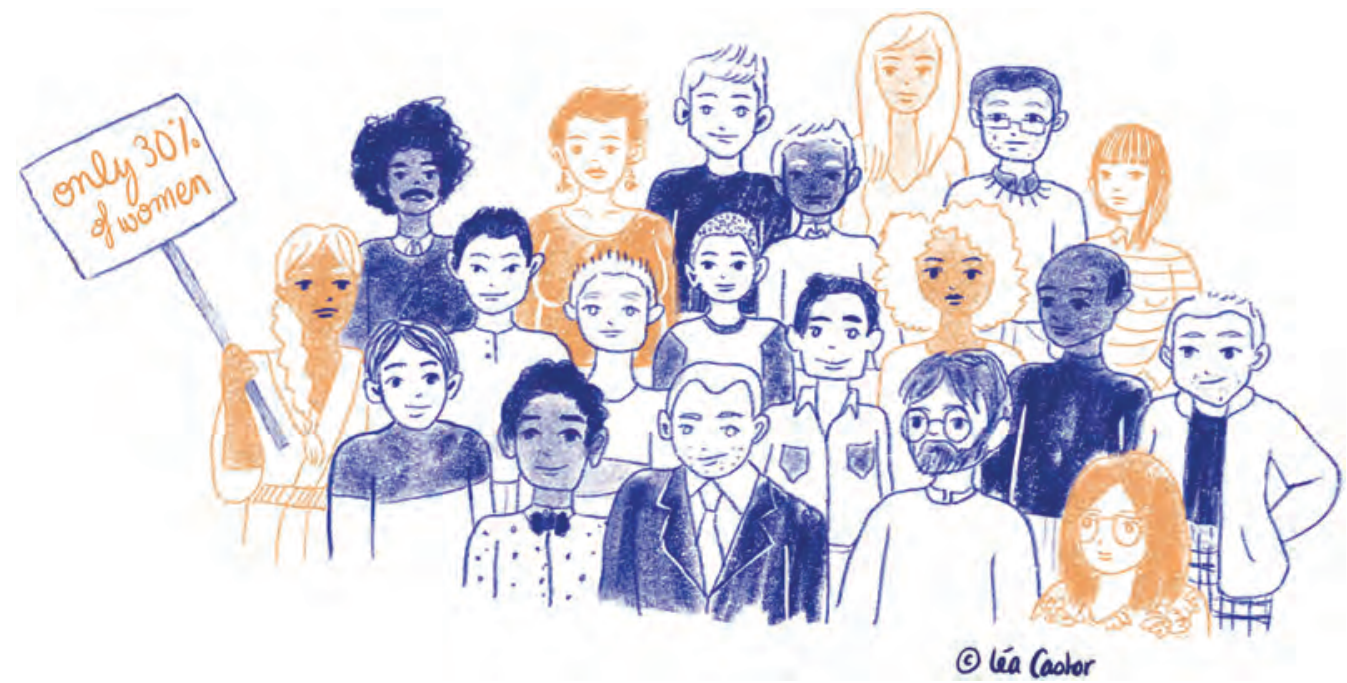

Dazu habe ich natürlich meine persönliche Meinung, aber da Sie mich als CWM-Vorsitzende interviewen und diese Frage sehr kontrovers diskutiert wird, ziehe ich es vor, sie nicht zu beantworten. Viele Wissenschaftlerinnen, aber auch Männer guten Willens, befürchten, dass eine Frauenquote am Ende negative Folgen haben und Stereotype und Diskriminierung verstärken könnte, während andere meinen, dass sie sich für eine größere Vielfalt in der Wissenschaft als sehr nützlich erweisen kann. Die Relevanz der Quote hängt in jedem Fall von der spezifischen Situation in jedem Land und jeder Organisation ab.

Wenn Sie einen Wunsch frei hätten, die Geschlechterlücke in der Mathematik zu schließen, welcher wäre das?

Das Thema, das für den Internationalen Tag der Mathematik 2021 gewählt wurde, „Mathematik für eine bessere Welt", spricht wirklich mein Herz und meinen Verstand an. Wir brauchen ein spezifisches Bewusstsein und Anstrengungen, um die Geschlechterkluft in der Mathematik zu schließen, aber diese Sache ist Teil eines viel globaleren Ziels, nämlich der Entwicklung der Mathematik als Beitrag zur Nachhaltigkeit der menschlichen Zivilisation und des Lebens auf unserem schönen Planeten. Ich beziehe mich sehr oft auf die 17 von den Vereinten Nationen definierten Ziele für nachhaltige Entwicklung, und eines davon, das Ziel Nr. 5, ist die Gleichstellung der Geschlechter. Dort heißt es:

Die Gleichstellung der Geschlechter ist nicht nur ein grundlegendes Menschenrecht, sondern eine notwendige Grundlage für eine friedliche, wohlhabende und nachhaltige Welt.

Ich wünsche mir, dass dies von Mathematikerinnen und Mathematikern weltweit umfassend und in all seiner Tiefe verstanden wird. Denn ich denke, dass die Geschlechterlücke in der Mathematik nur über ein breit angelegtes Verständnis verkleinert werden kann.

\section{Projektwebseite}

[1] Gender Gap In Science. A Global Approach to the Gender Gap in Mathematical, Computing, and Natural Sciences: How to Measure It, How to Reduce It? gender-gap-in-science.org/project/

Illustrationen: Léa Castor

Übersetzung aus dem Englischen von Marc Oprisiu und Thomas Vogt, DMV-Medienbüro

\begin{abstract}
Marie-Françoise Roy, seit 1985 Professorin für Mathematik an der Universität Rennes in Frankreich und mittlerweile emeritiert, engagiert sich seit Jahrzehnten für Frauen in der Mathematik: Sie war eine der Gründerinnen und erste Präsidentin von Femmes et Mathématiques (1987), Mitgründerin von European Women in Mathematics (EWM) und deren Vorsitzende (convenor 2009-2013). Seit 2015 ist sie Vorsitzende des Komitees für Frauen in der Mathematik der IMU, kurz CWM.

Von 2004 bis 2007 war sie Präsidentin der Société Mathématique de France (SMF).
\end{abstract}

Thomas Vogt, Medienbüro Mathematik,

FU Berlin, Institut für Mathematik, Königin-Luise-Straße 24-26, 14195 Berlin

medienbuero@mathematik.de 